\title{
Medial olivocochlear suppression in musicians versus non-musicians
}

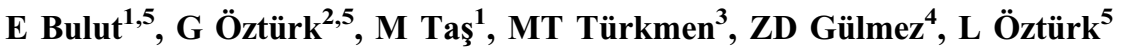 \\ ${ }^{1}$ Department of Audiology, Faculty of Health Sciences, Trakya University, Edirne, Turkey \\ ${ }^{2}$ Department of Physiotherapy and Rehabilitation, Faculty of Health Sciences, Trakya University, Edirne, Turkey \\ ${ }^{3}$ Department of Otorhinolaryngology, Faculty of Medicine, Trakya University, Edirne, Turkey \\ ${ }^{4}$ Department of Audiology, Faculty of Health Sciences, Istanbul University, Istanbul, Turkey \\ ${ }^{5}$ Department of Physiology, Faculty of Medicine, Trakya University, Edirne, Turkey
}

Received: May 7, 2018

Accepted: May 11, 2019

\begin{abstract}
The medial olivocochlear efferent (MOCE) branch synapses with outer hair cells (OHCs), and the efferent pathway can be activated via a contralateral acoustic stimulus (CAS). The activation of MOCE can change OHC motile responses and convert signals that are capable of controlling the sensitivity of the peripheral hearing system in a frequency-specific manner. The aim of this study was to examine the MOCE system activity in professional musicians using transient evoked otoacoustic emission test and CAS. Musician group showed stronger suppression in all frequency bands in the presence of CAS.
\end{abstract}

Keywords: contralateral acoustic stimulus, broadband noise, medial olivocochlear efferent system, otoacoustic emission, cochlear micromechanics

\section{Introduction}

Olivocochlear efferents help to enhance detection of signals in background noise by hyperpolarizing outer hair cells (OHCs) and reducing basilar membrane motion $(6,13)$. Efferent neurons in mammals are divided into lateral and medial bundles, which also differ in function (14). Lateral olivocochlear efferents originate from lateral areas of superior olivary complex and send thin unmyelinated axons to dendrites of afferent fibers beneath the cochlear inner hair cells. Medial olivocochlear efferents (MOCEs) originate from more medial and rostal areas of the superior olivary complex and send thicker and myelinated axons to OHCs $(13,14)$. When activated, MOCEs can change cochlear sensitivity in $10 \mathrm{~ms}$ (fast modulation) or in $10 \mathrm{~s}$ (slow modulation) (30).

Cochlear micromechanics are regulated by MOCE system through affecting contractility of $\mathrm{OHCs}(12,14,15)$. OHCs may elongate and shorten (contractility) in response to intracellular potential changes and these properties of OHCs are responsible for amplification of vibrations induced by acoustic stimuli $(1,28)$. Contractility of OHCs appears to amplify basilar membrane vibrations actively, a process that is often referred to as cochlear amplification (1). An ATP-independent cellular electromotility mechanism based on the activity of prestin is responsible for $\mathrm{OHC}$ contractility $(4,11,32)$.

Corresponding author: Dr. Erdogan Bulut, $\mathrm{PhD}$

Department of Audiology, Faculty of Health Sciences, Trakya University

22030 Edirne, Turkey

Phone: +90 28421330 42; Fax: +90 28421261 07; E-mail: erdoganbulut@trakya.edu.tr 
Prestin is a transmembrane motor protein described in the lateral wall of OHCs. Prestin uses cytoplasmic anions as extrinsic voltage sensors and changes $\mathrm{OHC}$ length in response to membrane potential alterations (9). In a recent study, prestin-based OHC motility has been demonstrated to be necessary for mammalian cochlear amplification (10).

Another result of $\mathrm{OHC}$ contractility is production of sounds within cochlea. These sounds (27) are called otoacoustic emissions (OAEs). OAEs are sensitive to activation of the MOCE. In response to MOCE activation, the amplitudes of OAEs are decreased or suppressed $(2,13)$. The typical way to measure suppression is to first measure the OAE in the ipsilateral ear in the absence of contralateral acoustic stimulus (CAS). Then, the OAE is measured again in the ipsilateral ear but with CAS. The CAS activates the MOCE and alters the OAE. The absence of suppression is a pathological event, which may predict efferent auditory system dysfunction $(16,17)$. In professional musicians, auditory perceptual processes including auditory skills, frequency discrimination, auditory memory, and auditory attention show remarkable differences when compared to non-musicians (25). Perrot et al. (25) demonstrated a stronger bilateral cochlear suppression in musicians versus nonmusicians. Brashears et al. (5) demonstrated increases in the activation of MOCE system and the middle ear muscles reflex thresholds in musicians. The neural basis of higher auditory perceptual performance in musicians has not yet been fully explained (24). The aim of this study was to compare MOCE suppression in professional musicians versus non-musicians using transient evoked OAEs (TEOAEs) and CAS.

\section{Material and Methods}

\section{Subjects}

The study was designed and performed according to the Declaration of Helsinki. After obtaining ethical approval and written informed consent forms, 26 professional musicians and 17 non-musician control subjects were included in the study. The musicians were volunteers from the Balkan Symphonic Orchestra who had been playing an instrument for at least 5 years. Volunteers who had tinnitus, middle ear or external ear canal pathologies, or a history of noise (other than music) exposure were excluded. All participants underwent audiometry (between 0.5 and $8 \mathrm{kHz}$ ), tympanometry [226 Hz at $85 \mathrm{~dB}$ sound pressure level (SPL)], and contralateral acoustic reflex test (between 0.5 and $4 \mathrm{kHz}$ ). TEOAEs were measured in the ipsilateral ear with and without CAS.

Ethics committee approval for this study was received from the ethics committee of Trakya University Faculty of Medicine Ethics Committee of Noninvasive Clinical Researches (approval no.: TÜTF-GOAEK 2014/54).

\section{Audiological evaluation}

Pure tone audiometry was conducted using AC-40 Clinical Audiometers (Interacoustic Audiometer AZ-7, Middelfart, Denmark). Measurements were taken in a soundproof room with TDH-39 (Telephonics, NY, USA) earphones. Hearing thresholds were determined for octave frequencies between 0.5 and $8 \mathrm{kHz}$. Middle-ear pathologies and stapes or acoustic reflexes were assessed by impedance audiometer (Interacoustic Audiometer AZ-7) and a recording device (XYT Recorder AG-3, Middelfart, Denmark). Acoustic reflex thresholds were determined for octave frequencies between 0.5 and $4 \mathrm{kHz}$. 


\section{TEOAE recording and contralateral acoustic stimulation}

TEOAEs with or without CAS were measured using an Otodynamics ILO 292 USB II model OAE instrument (Otodynamics, Hatfield, UK). Proper-sized adult probes that ensure a tight seal in the ear canal were used. The probe was calibrated using the $1 \mathrm{cc}$ acoustic calibration cavity. Broadband noise was used as the CAS. Test probes were placed into both ear canals and linear transient-type 260 sweeps of $80 \mathrm{~dB}$ peSPL clicks were delivered to the ipsilateral ear at a rate of 50/s, while broadband noise at $60 \mathrm{~dB}$ SPL was presented to the contralateral ear. TEOAEs in ipsilateral ear, obtained both with and without contralateral broadband noise, were recorded. Two hundred sixty accepted sweeps were obtained and a noise rejection level at $45 \mathrm{~dB}$ SPL was used. Emission and noise amplitudes were calculated by a software Otodynamics ILO 292 USB II (Hatfield, UK) in half-octave frequency bands centered at 1, $1.4,2,2.8$, and $4 \mathrm{kHz}$. TEOAE amplitudes and signal/noise ratios (SNR) that were obtained by each stimulus at $1,1.4,2,2.8$, and $4 \mathrm{kHz}$ were used as study parameters. Suppression was determined by subtracting the TEOAE amplitudes with CAS from the TEOAE amplitudes without CAS. Suppression was calculated for each frequency range.

\section{Statistical analysis}

Statistical analysis was carried out using SPSS 15.0 for Windows (IBM Corporation, NY, USA). The normality of TEOAE amplitudes was analyzed by Kolmogorov-Smirnov $Z$ analysis. TEOAE amplitudes in the absence and presence of contralateral broadband noise were compared with Wilcoxon test or Mann-Whitney $U$ test. The value of $p \leq 0.05$ was considered to be significant. As the data were not convenient for parametric tests, intragroup comparisons were performed using Wilcoxon test, whereas between-group comparisons were performed using Mann-Whitney $U$ test.

\section{Results}

The musician group consisted of 14 (54\%) female and 12 (46\%) male subjects. The mean age of study group was $34.3 \pm 1.4$ years (range: 19-46 years). The non-musician group [mean age: $37.7 \pm 4.8$ years; male/female: $7(41.2 \%) / 10(58.8 \%)$ ] was comparable to musician group in terms of age and gender ( $p>0.05$ for both).

\section{Audiologic data}

All participants had air-conduction hearing threshold of better than $15 \mathrm{~dB}$ between 250 and $8,000 \mathrm{~Hz}$. They had less than $10 \mathrm{~dB}$ air-bone gap between 500 and 4,000 Hz. Normal type tympanogram (type "A") in tympanometry test was obtained; stapes reflex thresholds were given in Table I. Pure tone audiogram and stapes reflex threshold results were comparable between musician and non-musician groups.

\section{TEOAE responses and suppression}

An SNR higher than $3 \mathrm{~dB}$ in the suboctave frequency bands between 1 and $4 \mathrm{kHz}$ was treated as a pass for TEOAE. TEOAE responses were similar in both right and left ears $(p>0.05)$.

When TEOAE amplitudes were compared in both the presence and absence of CAS, significant differences were observed in 1, 1.4, 2, 2.8, and $4 \mathrm{kHz}$ center frequencies $(p<0.05)$ in the musician group (Fig. 1), whereas there was no significant difference in 1, 1.4, 2, 2.8 , and $4 \mathrm{kHz}$ center frequencies $(p>0.05)$ in the non-musician group (Fig. 2). 
Table I. Contralateral stapes reflex thresholds with sound stimulus at different frequencies

\begin{tabular}{|l|c|c|}
\hline Stapes reflex threshold & Musicians $(\boldsymbol{n}=\mathbf{2 6})^{*}$ & Non-musicians $(\boldsymbol{n}=\mathbf{1 7})$ \\
\hline dB SPL at $0.5 \mathrm{kHz}$ & $92.7 \pm 5.7$ & $92.5 \pm 6.1$ \\
\hline dB SPL at $1 \mathrm{kHz}$ & $92.9 \pm 5.4$ & $93.4 \pm 6.2$ \\
\hline dB SPL at $2 \mathrm{kHz}$ & $92.0 \pm 6.1$ & $92.9 \pm 6.4$ \\
\hline dB SPL at $4 \mathrm{kHz}$ & $92.0 \pm 5.4$ & $92.0 \pm 6.6$ \\
\hline
\end{tabular}

dB: decibel; SPL: sound pressure level; kHz: kilohertz.

*All comparisons revealed comparable stapes reflex thresholds in both musicians and non-musicians

TEOAE Amplitude (Musicians)

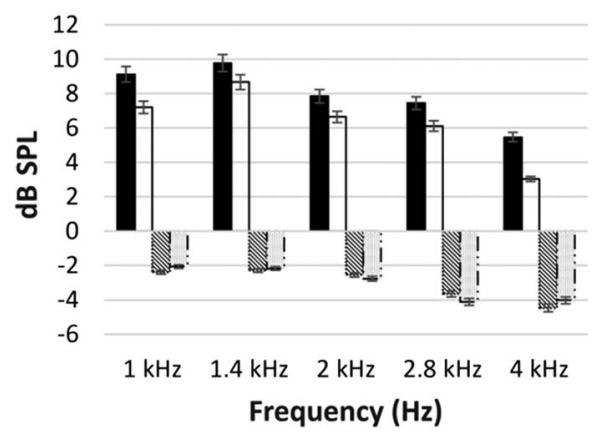

TEOAE Amplitude (NonMusicians)

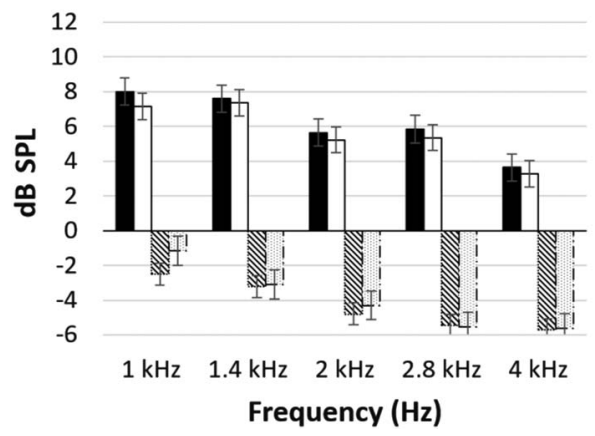

no-CAS / signal

口CAS / signal

$\mathbb{N}$ no-CAS / noise

r.CAS / noise

- no-CAS / signal

$\square$ CAS / signal

no-CAS/ noise

- CAS / noise
Fig. 1. TEOAE amplitudes in the absence and presence of contralateral stimulus in the musician group
Fig. 2. TEOAE amplitudes in the absence and presence of contralateral stimulus in the non-musician group

MOCE suppression effect in musicians was more evident in all frequency bands (Table II). We also compared SNRs in the presence and absence of CAS and found significant difference between 1, 2, 2.8, and $4 \mathrm{kHz}(p<0.05)$ center frequencies in the musicians (Fig. 3). On the other hand, significant difference was observed only in $1 \mathrm{kHz}$ center frequency $(p=0.001)$ in the non-musician group (Fig. 4). 
Table II. TEOAE suppression values (dB SPL)* in musicians and non-musicians

\begin{tabular}{|l|c|c|c|}
\hline Frequency $(\mathbf{k H z})$ & Musicians $(\boldsymbol{n}=\mathbf{2 6})$ & Non-musicians $(\boldsymbol{n}=\mathbf{1 7})$ & $\boldsymbol{p}$ \\
\hline 1 & $1.92 \pm 0.26$ & $0.84 \pm 0.31$ & 0.02 \\
\hline 1.4 & $1.11 \pm 0.17$ & $0.24 \pm 0.32$ & 0.02 \\
\hline 2 & $1.20 \pm 0.22$ & $0.42 \pm 0.09$ & 0.02 \\
\hline 2.8 & $1.33 \pm 0.29$ & $0.49 \pm 0.15$ & 0.14 \\
\hline 4 & $2.44 \pm 0.60$ & $0.35 \pm 0.16$ & 0.04 \\
\hline
\end{tabular}

dB: decibel; SPL: sound pressure level; kHz: kilohertz.

*All data given are mean \pm SEM

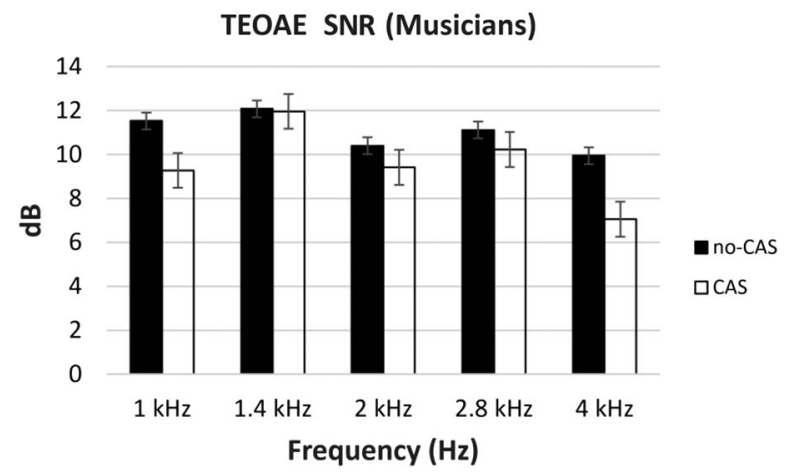

Fig. 3. SNRs of TEOAE in the musician group

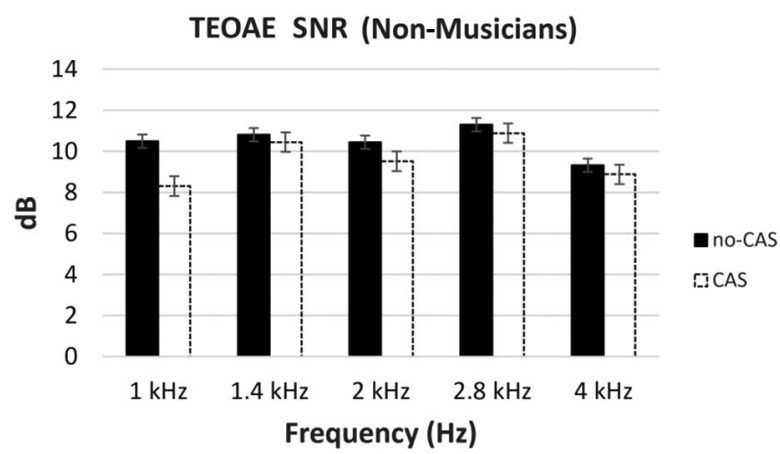

Fig. 4. SNRs of TEOAE in the non-musician group

\section{Discussion}

Sound enters the cochlea through the outer and middle ear and is analyzed for hydromechanical frequency discrimination along the length of the cochlea in the inner ear. For hydromechanical analysis, at each cochlear segment, OHCs amplify basilar membrane motion which is called "cochlear amplification" $(7,8,18,19)$. MOCEs terminate on OHCs and modify $\mathrm{OHC}$ action, which in turn controls the gain of cochlear amplifier (14). OAEs are produced by basilar membrane motion, which is created by the energy sent back as a result of 
reflection and distortion mechanisms (29). MOCEs lead to mechanical changes, affect basilar membrane motion, and eventually change OAEs. The amplitude of OAEs recorded from one ear can be altered by a sound presented in the contralateral ear. This is known as soundinduced suppression of OAEs, as the amplitudes are reduced $(3,22,31)$. This suppression provides a non-invasive tool to evaluate the function of this auditory efferent system (3). We evaluated frequency-specific MOCE acoustic reflex obtained by the CAS (broadband noise $60 \mathrm{~dB}$ SPL) in TEOAE records.

The MOCE acoustic reflex is cpnsidered to provide frequency-specific feedback that adjusts the gain of cochlear amplifier, but little is known about the function of the frequencyspecific reflex (20). On the other hand, fine auditory sensitivity requires cochlear micromechanics, which are modulated by MOCE. In addition, auditory perception may be facilitated by an increase in the activity of MOCE. A sharpened auditory perception may play an important role in competitive musical environments (24). Several studies $(5,21,23,25)$ have found that efferent suppression was stronger in musicians than non-musicians, due to the activity of MOCEs. Interestingly, Perrot and Collet (24) noted that musicians have stronger subcortical responses to speech stimuli in background noise than non-musicians. In parallel to those findings, we found a stronger suppression in the musician group than in non-musicians at all frequency bands. We used both amplitudes and SNR responses, whereas previous studies used only amplitudes to evaluate the OHC motile responses.

Some studies provided a direct evidence of a functional olivocochlear pathway, which modulates contralateral cochlear micromechanics, through the MOCE system $(24,26)$. MOCE fibers synapse on OHCs, and activation of these fibers inhibits basilar membrane responses to low-level sounds (13). This MOCE-induced decrease in the gain of cochlear amplifier is reflected in OAE changes. Any OAE can be used to monitor effect of MOCE on the cochlear amplification $(6,13)$. In this study, we observed suppression of TEOAE amplitudes and SNR values in the musician group. This suppression may stem from elevated $\mathrm{OHC}$ motile responses, which may contribute to better pitch perception in musicians. In the non-musician group, there was no suppression of TEOAE amplitudes but SNR values were suppressed only at $1 \mathrm{kHz}$, which is in the human speech frequency range. These results suggest that the activity of MOCE system may be adopted to monitor hearing deficits or alterations in musicians.

\section{Acknowledgements}

This study was presented to be a presentation at the 41st National Physiology Congress Turkish Society of Physiological Sciences Congress, Çanakkale, Turkey, May 27-30th, 2015 and published in the abstract form [Supplement issue S704 (215): 94-95 September 2015] in the Acta Physiologica.

\section{Conflict of interest}

The authors declare no conflict of interest.

\section{REFERENCES}

1. Ashmore JF, Mammano F: Can you still see the cochlea for the molecules? Curr. Opin. Neurobiol. 11(4), 449-454 (2001)

2. Bargones JY, Burns EM: Suppression tuning curves for spontaneous otoacoustic emissions in infants and adults. J. Acoust. Soc. Am. 83(5), 1809-1816 (1988)

3. Berlin CI, Hood LJ, Wen H, Szabo P, Cecola RP, Rigby P, Jackson DF: Contralateral suppression of non-linear click-evoked otoacoustic emissions. Hear. Res. 71(1-2), 1-11 (1993) 
4. Bray PJ (1989): Clicked evoked otoacoustic emissions and the development of a clinical otoacoustic hearing test instrument [Dissertation]. London University, London

5. Brashears SM, Morlet TG, Berlin CI, Hood LJ: Olivocochlear efferent suppression in classical musicians. J. Am. Acad. Audiol. 14(6), 314-324 (2003)

6. Cooper NP, Guinan JJ, Jr: Efferent-mediated control of basilar membrane motion. J. Physiol. 576(Pt. 1), 49-54 (2006)

7. Dallos P: Active cochlea. J. Neurosci. 12(12), 4575-4585 (1992)

8. Dallos P: Cochlear amplification, outer hair cells and prestin. Curr. Opin. Neurobiol. 18(4), 370-376 (2008)

9. Dallos P, Falkler B: Prestin, a new type of motor protein. Nat. Rev. Mol. Cell Biol. 3(2), 104-111 (2002)

10. Dallos P, Wu X, Cheatham MA, Gao J, Zheng J, Anderson CT, Jia S, Wang X, Cheng WH, Sengupta S, He DZ, Zuo J: Prestin-based outer hair cell motility is necessary for mammalian cochlear amplification. Neuron. 58(3), 333-339 (2008)

11. Frolenkov GI, Mammano F, Kachar B: Regulation of outer hair cell cytoskeletal stiffness by intracellular $\mathrm{Ca}^{2+}$ : underlying mechanism and implications for cochlear mechanics. Cell Calcium. 33(3), 185-195 (2003)

12. Groff JA, Liberman MC: Modulation of cochlear afferent response by the lateral olivocochlear system: activation via electrical stimulation of the inferior colliculus. J. Neurophysiol. 90(5), 3178-3200 (2003)

13. Guinan JJ, Jr: Olivocochlear efferents: anatomy, physiology, function, and the measurement of efferent effects in humans. Ear Hear. 27(6), 589-607 (2006)

14. Guinan JJ, Jr (1996): Physiology of Olivocochlear efferents. In: The Cochlea, eds Dallos P, Popper AN, Fay RR, Springer, New York, pp. 435-502

15. Guinan JJ, Jr, Gifford ML: Effects of electrical stimulation of efferent olivocochlear neurons on cat auditorynerve fibers. III. Tuning curves and thresholds at CF. Hear. Res. 37(1), 29-45 (1988)

16. Hood LJ, Berlin CI, Bordelon J, Rose K: Patients with auditory neuropathy/dys-synchrony lack efferent suppression of transient evoked otoacoustic emissions. J. Am. Acad. Audiol. 14(6), 302-313 (2003)

17. Hood LJ, Berlin CI, Hurley A, Wen H (1996): Suppression of otoacoustic emissions in normal hearing individuals. In: Hair Cells and Hearing Aids, ed Berlin CI, Singular Publishing, London, 1st ed., pp. 57-72

18. Kemp DT: Stimulated acoustic emissions from within the human auditory system. J. Acoust. Soc. Am. 64(5), 1386-1391 (1978)

19. Liberman MC, Gao J, He DZ, Wu X, Jia S, Zuo J: Prestin is required for electromotility of the outer hair cell and for the cochlear amplifier. Nature 419(6904), 300-304 (2002)

20. Lilaonitkul W, Guinan, JJ, Jr: Frequency tuning of medial-olivocochlear-efferent acoustic reflexes in humans as functions of probe frequency. J. Neurophysiol. 107(6), 1598-1611 (2012)

21. Micheyl C, Khalfa S, Perrot X, Collet L: Difference in cochlear efferent activity between musicians and non-musicians. Neuroreport 8(4), 1047-1050 (1997)

22. Mott JB, Norton SJ, Neely ST, Warr WB: Changes in spontaneous otoacoustic emissions produced by acoustic stimulation of the contralateral ear. Hear. Res. 38(3), 229-242 (1989)

23. Parbery-Clark A, Skoe E, Kraus N: Musical experience limits the degradative effects of background noise on the neural processing of sound. J. Neurosci. 29(45), 14100-14107 (2009)

24. Perrot X, Collet L: Function and plasticity of the medial olivocochlear system in musicians: a review. Hear. Res. 308, 27-40 (2014)

25. Perrot X, Micheyl C, Khalfa S, Collet L: Stronger bilateral efferent influences on cochlear biomechanical activity in musicians than in non-musicians. Neurosci. Lett. 262(3), 167-170 (1999)

26. Perrot X, Ryvlin P, Isnard J, Guénot M, Catenoix H, Fischer C, Mauguière F, Collet L: Evidence for corticofugal modulation of peripheral auditory activity in humans. Cereb. Cortex. 16(7), 941-948 (2006)

27. Probst R, Lonsbury-Martin BL, Martin GK: A review of otoacoustic emissions. J. Acoust. Soc. Am. 89(5), 2027-2067 (1991)

28. Robles L, Ruggero MA: Mechanics of the mammalian cochlea. Physiol. Rev. 81(3), 1305-1352 (2001)

29. Shera CA, Guinan JJ: Evoked otoacoustic emissions arise by two fundamentally different mechanisms: a taxonomy for mammalian OAEs. J. Acoust. Soc. Am. 105(2), 782-798 (1999)

30. Sridhar TS, Liberman MC, Brown MC, Sewell WF: A novel cholinergic "slow effect" of efferent stimulation on cochlear potentials in the guinea pig. J. Neurosci. 15(5 Pt. 1), 3667-3678 (1995)

31. Zhao W, Dhar S: The effect of contralateral acoustic stimulation on spontaneous otoacoustic emissions. J. Assoc. Res. Otolaryngol. 11(1), 53-67 (2010)

32. Zheng J, Shen W, He DZ, Long KB, Madison LD, Dallos P: Prestin is the motor protein of cochlear outer hair cells. Nature 405(6783), 149-155 (2000) 